\title{
El acceso a la justicia de la población campesino-indígena: Un estudio en el Consultorio Jurídico gratuito de la Universidad Católica de Cuenca, extensión Cañar
}

\section{The access to the justice of the peasant-indigenous population:A survey in the free Legal Office of the Catholic University of Cuenca, extensión Cañar.}

\author{
Correa, Wilson* \& Andrade, Hernan \\ Carrera de Administración de Empresas y Derecho,Universidad Católica de Cuenca \\ Cañar, 030201, Ecuador \\ *wbcorreac@ucacue.edu.ec
}

\begin{abstract}
Resumen
Este estudio investigativo realiza un análisis retrospectivo en el periodo 2013-2015, durante el cual se implementó de forma concreta el proyecto de vinculación con la sociedad: Consultorio Jurídico gratuito de la Universidad Católica de Cuenca, en la extensión Cañar. El objetivo principal de este trabajo es analizar cómo su gestión ha contribuido a garantizar el derecho de acceso a la justicia en los sectores vulnerables y de bajos recursos económicos del cantón Cañar. Esto ha ido transformando realidades de exclusión y discriminación en el marco de los enfoques de los derechos humanos, de género y de interculturalidad; de principios, estándares y lineamientos de igualdad-equidad y el de vinculación con la comunidad aplicable a la educación superior en Ecuador. Para ello se realizó una investigación descriptiva-exploratoria, teniendo como muestra poblacional a los usuarios atendidos en elperiodo de referencia, y sobre la base de los registros de atención del consultorio. Elestudio comprende tres momentos: a) recoleccióny evaluación de datos; b) fundamentación teórica; c) tabulación delainformación y definición de indicadores sociales y de gestión. Los resultados permitieron concluir que el Consultorio Jurídicose ha constituido en alternativa de asesoría y patrocinio qeu fortalece los procesos de acceso a la justicia para quienes acuden en búsqueda de restitución de sus derechos, en particular de mujeres campesino-indígenas pobres del área rural del cantón, tantoporel carácter gratuito de la atención como por la eficiencia reflejada en el número de los acasos atendiddos y resueltos.
\end{abstract}

Palabras clave: Acceso a la justicia, derechos humanos, discriminación, género, interculturalidad, vinculación con la comunidad.

\begin{abstract}
This research study carries out a retrospective analysis in the period (2013-2015) during which the proyect of linkage to society was implemented in a concrete way: Free of charge Legal Office of the Catholic University of Cuenca, Cañar extension. The main objective of this work is to analyze the way in which its management has contributed to guarantee the right to access justice in the vulnerable and low economic resource sectors of the Cañar canton, which has transformed realities of exclusion and discrimination, within the human rights, gender and intercultural frameworks, as well as of principles, standards and guidelines of equality-equity and that of linkage to the community applicable to higher education in Ecuador. For this purpose, a descriptive-exploratory research was carried out having as population sample the users attendedin the refered period and on the bases of the legal office records. The study includes three moments: a) data collection and evaluation; $b$ ) theoretical foundation; $c$ ) information tabulation and definition of social and management indicators. The results allow to conclude that, the Legal Office, has been constituded as an advice and sponsorship alternative that strengthens the processes of acces to justice for those who seek the restitution of their rights, paticularly of poor peasant-indigenous women in rural area of the Canton, both for the free nature of the services and for the efficiency reflected in the number of the cases attended and resolved.
\end{abstract}

Key words: Access to the justice, human rights, discrimination, gender, intercultural, entailment with the community . 


\section{Introducción}

En el Ecuador persisten deficiencias para facilitar el acceso a la justicia y garantizar el derecho a la defensa, no todos los ciudadanos cuentan con los medios y circunstancias para defenderse, tanto la defensoría pública como la defensoría privada atraviesan una situación similar debido a que los defensores no están debidamente capacitados, el comportamiento ético de los abogados no ha sido el más adecuado, no hay responsabilidad para atender debidamente los casos que se asignan, primando una suerte de explotación económica; esto ha afectado el acceso a la justicia de las personas que socialmente se encuentran en desventaja por su desconocimiento, situación geográfica, etnia, género, condición económica; al no poder acceder a los servicios de defensa legal para la protección de sus derechos.

Esta problemática no ha sido diferente en el cantón Cañar, al encontrarse entre los sectores más marginados y pobres del país con población mayoritariamente rural, los problemas de acceso a la justicia ordinaria en particular de la mujer rural han sido permanentes debido a patrones de exclusión y desigualdad étnica.

Frente a esta situación la Universidad Católica de Cuenca entidad de educación superior en el año 2007 implementa el "Consultorio Jurídico gratuito" de la facultad de derecho, mediante resolución del H. Consejo Directivo y amparada en el artículo 193 de la Constitución de la república y los artículos 286 y 293 del Código Orgánico de la función judicial; y en agosto 28 de año 2013 obtiene la autorización de acuerdo con resolución No DP-CPA-2013017 del Comité de Patrocinio y Acreditación.

El objetivo del presente trabajo es demostrar como la implementación de un sistema de defensoría gratuita de la Universidad, a través del patrocinio jurídico y de la asesoría, ha contribuido a mejorar el acceso a la justicia y garantizar los derechos de la población del área rural, en su mayoría mujeres campesino-indígenas del cantón Cañar y sus parroquias desde el año 2013 al 2015, fortaleciendo los procesos de construcción de igualdad y la transformación de prácticas sociales desde la educación superior.

En este contexto, el estudio se inscribe en el marco de los diferentes instrumentos internacionales como la Declaración Universal de los Derechos Humanos (1948); la Convención de los Derechos Políticos de las Mujeres (1952); el Pacto Internacional de los Derechos Civiles y Políticos (1966).

El documento contiene elementos conceptuales sobre el "acceso a la justicia" desde una perspectiva de los derechos humanos, de género e interculturalidad, así como de los principios, estándares y lineamientos propuestos por la Secretaría de Educación Superior, Ciencia, Tecnología e Innovación (Senescyt) aplicable a la temática tratada; un segundo componente se refiere a la metodología aplicada en la investigación, la población objeto de estudio y el proceso aplicado para la recolección de datos; posteriormente se presentan los resultados en función del análisis de cuadros estadísticos, gráficos e indicadores; para finalmente establecer conclusiones y recomendaciones.

\section{Marco Teórico}

\subsection{Definiciones y aspectos generales}

Para una mejor comprensión de la temática se analizan varios enfoques y principios sobre el "acceso a la justicia" como derecho humano fundamental en un sistema democrático, adicionalmente se fundamenta en estándares y lineamientos propuestos por la Senescyt para la construcción de igualdad desde la educación superior.

\subsection{Enfoques para abordar el acceso a la justicia.}

Las desigualdades en el acceso a la justicia se manifiestan en distintas formas, según las condiciones sociales, culturales y económicas de las sociedades.

El marco conceptual para tratar la problemática de "acceso a la justicia", tendrá como base tres enfoques:

El primero es el enfoque de derechos humanos fundamentado en el discurso global de derechos; como valores vinculados a la dignidad, la libertad, la igualdad de las personas y las garantías y obligaciones del estado, un segundo enfoque es el de género el cual permite entender las relaciones construidas socialmente entre hombres y mujeres; y el de interculturalidad entendido en términos amplios como el contacto e intercambio entre culturas en condiciones de igualdad. (Walsh, 2009)

A continuación se revisan definiciones de conceptos respecto de estos enfoques:

\subsubsection{El enfoque de derechos humanos}

"derechos humanos son aquellos derechos-civiles y políticos, económicos, sociales y culturales- inherentes a la persona humana, así como aquellas condiciones y situaciones indispensables, reconocidas por el Estado a todos sus habitantes sin ningún tipo de discriminación, para lograr un proyecto de vida digna". (de Derechos Humanos, 2011)

\subsubsection{Enfoque de género}

“... el género se concibe como un elemento constitutivo de las relaciones sociales basado en las diferencias que se perciben entre los sexos; y es una manera primaria de significar las relaciones de poder". (Scott, 1993)

\subsubsection{Enfoque de interculturalidad}

“el reconocimiento, valorización y fortalecimiento de la identidad y enfatiza la diversidad, ya no como una barrera sino como un recurso; en otras palabras la unidad en la diversidad”. (Moya, 1999) 


\subsection{El acceso a la justicia}

Un primer elemento a tomar en cuenta en el acceso a la justicia como derecho colectivo es la existencia de barreras económicas, por cuanto no todos los ciudadanos cuentan con los recursos para defenderse, lo cual se refleja en el desigual tratamiento de los casos; un segundo elemento tiene que ver con las barreras culturales y de género que inciden en las condiciones de desigualdad social e inequidad, justamente el desconocimiento del derecho; hace que el primer escalón de acceso a la justicia sea la representación jurídica eficiente en un conflicto que garantice igualdad de condiciones.

Al interior del enfoque de derechos la Comisión Interamericana de Derechos Humanos (CIDH), define al acceso a la justicia como:

"Una respuesta judicial efectiva (...) comprende la obligación de hacer accesibles recursos judiciales sencillos, rápidos, idóneos e imparciales de manera no discriminatoria, para investigar sancionar y reprimir estos actos, y prevenir de esta manera la impunidad". (de Derechos Humanos, 2007)

Se entiende entonces el acceso a la justicia como la posibilidad de que todas las personas sin distinción alguna puedan utilizar los servicios jurídicos del estado con el fin de resarcir daños ocasionados y reclamar sus derechos.

\subsection{Principios que sustentan el acceso a la justicia}

Los principios que sustentan la problemática de acceso a la justicia son: de igualdad y equidad entendido el primero como el reconocimiento de la igualdad en dignidad de todo ser humano y el segundo como el conjunto de medidas que contribuyen a modificar la situación actual de desigualdad; el principio de vinculación orgánica con la comunidad, comprendida como el establecimiento de una relación de aprendizaje mutuo entre las instituciones de educación superior y las comunidades en las cuales se encuentran insertas, superando la acción académica aislada de su contexto o planteada como asistencialista. (Secretaría de Educación Superior, 2015)

La implementación del proyecto de vinculación: Consultorio Jurídico Gratuito se fundamenta en los principios de vinculación orgánica con la comunidad y de igualdad y equidad definiéndose lineamientos que responden al eje de género e interculturalidad como se presenta en la tabla. 1

\section{Metodología}

\subsection{Tipo de Investigación}

La investigación es de tipo descriptiva-exploratoria con diseño de campo bibliográfico-documental, porque detalla los hechos observados a partir de la información de la propia institución para determinar la gestión realizada, sus particularidades y características.
Tabla 1

Fuente: Senescyt (Construyendo igualdad en la educación superior) Elaborado por: Los Autores

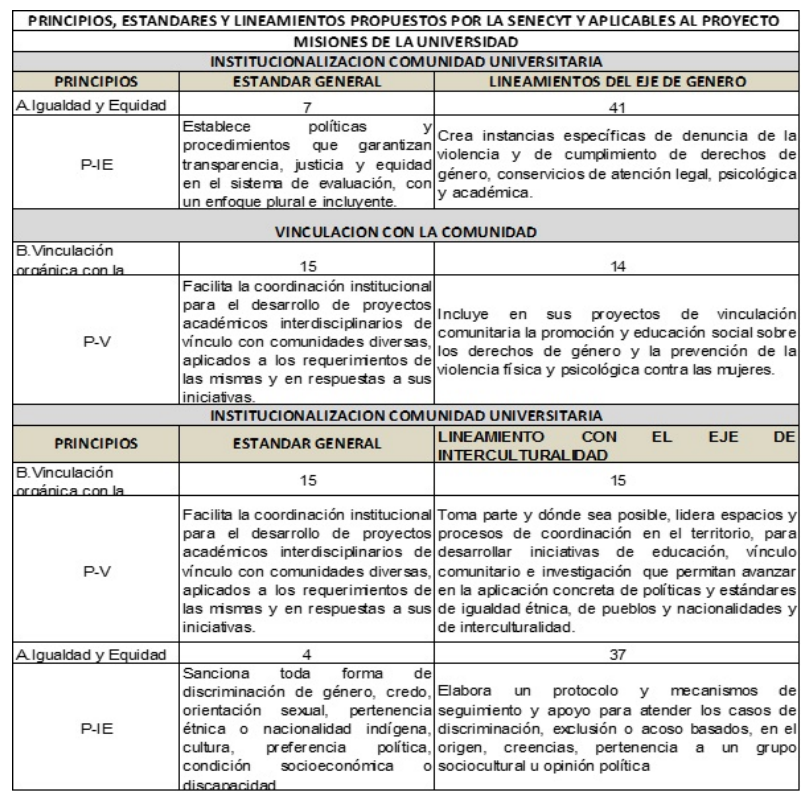

\subsection{Población}

La investigación se realizó en base a la información existente en el Consultorio Jurídico Gratuito de la Universidad Católica de Cuenca extensión Cañar, la población o universo está constituido por 1.077 usuarios existentes en los registros y que han asistido al consultorio en un período de tres años, clasificados en 942 procesos judiciales y 135 asesorías.

\subsection{Recolección de la información}

El enfoque aplicado para la recolección de datos es de carácter cuantitativo con una estrategia deductiva, recabada a través de fuentes secundarias como registros y fichas de usuarios existentes en los archivos de la institución; la información obtenida es objetiva respecto de varias características de la población y materia jurídica como son lugar de procedencia, sexo, etnia, estado civil, ocupación, nivel de instrucción, ingresos y discapacidad, área y estado del proceso.

Los datos fueros recogidos con el apoyo de los estudiantes de la carrera de derecho de cada uno de los registros de atención y revisados por el docente a cargo.

\subsection{Procesamiento de los datos recogidos}

Con la información recolectada se procedió a consolidar, tabular, organizar, construir indicadores y finalmente realizar un análisis estadístico que permitan evaluar la gestión del consultorio. 


\section{Resultados}

\subsection{Antecedentes}

El Consultorio Jurídico Gratuito de la facultad de derecho de la Universidad Católica de Cuenca extensión Cañar inicia a partir del año 2007 mediante resolución del H. Consejo Directivo y bajo convenio con el Gobierno Local, es el único con servicio social gratuito que presta este tipo de servicios en el cantón, los servicios legales están conformados por estudiantes y docentes juristas que a través del derecho buscan apoyar a grupos excluidos que acuden por necesidad de orientación y asesoramiento jurídico.

$\mathrm{Su}$ creación de acuerdo con el proyecto aprobado obedece al objetivo de: "brindar asesoría y patrocinio jurídico a través de la práctica efectiva y eficiente a los sectores vulnerables de la sociedad, garantizando el acceso a la justicia en igualdad de condiciones".

El Consultorio Jurídico está orientado de preferencia a personas de los estratos C y D (clasificación INEC) que son aquellos que no cuentan con recursos económicos y cuyo nivel de escolaridad es bajo.

\subsection{Componentes del estudio}

En función de los formularios de registro de usuarios se ha recabado información correspondiente a 3 años: desde enero del año 2013 hasta diciembre del año 2015, tiempo en el cual el proyecto ha logrado varios resultados tanto en su gestión como en el ámbito social en el cantón Cañar:

\subsubsection{Indicadores de Gestión relacionados con la aten- ción y servicio al usuario del Consultorio Jurídico}

a) Indicador de Cumplimiento y Satisfacción

Tabla 2

Fuente: Ficha de registro de usuarios atendidos en el Consultorio Jurídico Elaborado por: Loa Autores

INDICADOR DE CUMPLIMIENTOY SATISFACCION

\begin{tabular}{|l|c|c|c|c|}
\hline \multicolumn{1}{|c|}{ AÑOS } & 2013 & 2014 & 2015 & TOTAL \\
\hline PORCENTAJE DE CUMPLIMIENTO & $87 \%$ & $85 \%$ & $82 \%$ & $85 \%$ \\
\hline NUMERO DE PROCESOS TRAMITADOS Y GANADOS & 253 & 393 & 154 & 800 \\
\hline TOTAL DE PROCESOS ATENDIDOS ANUALMENTE & 291 & 464 & 187 & 942 \\
\hline
\end{tabular}

De la información recabada se desprende que de los 942 procesos judiciales en el período que va desde el 2013 al 2015 (no incluye asesorías) atendidos en el Consultorio Jurídico el $85 \%$ de los juicios han culminado con éxito (casos ganados), el $15 \%$ restante se reparte en 64 procesos perdidos, 44 casos han sido archivados, 13 se encuentran inactivos, y 15 han cambiado de defensor.

El comportamiento se presenta de manera similar durante todo el período de análisis, demostrando de esa manera que el indicador de cumplimiento es alto ( 9 de cada 10 procesos aproximadamente han culminado con éxito), y por ende se traduce en la satisfacción de los usuarios que acuden a este centro. b) Indicador de servicios que los usuarios utilizan por área de derecho

Tabla 3

Procesos ganados anualmente por área vs número total de procesos tramitados años.2013-2015 Fuente: Ficha de registro de usuarios atendidos en el Consultorio Jurídico Elaborado por: Los Autores

NDOCADOR DE SIRVICIOS CUE LOS USUARIOS UTIUZAN POR ARIA DI DIRTCHO

\begin{tabular}{|c|c|c|c|c|}
\hline AÑ̃os & 2013 & 2014 & 2015 & TOTAL \\
\hline CLASIFICACION POR AREA O MATERIA & \multicolumn{4}{|c|}{ PCACENTAIE DE CUMPLIMIENTO } \\
\hline FAMILIA MUIER, NINEEZ Y ADOLESCENCLA & $92 \%$ & $85 \%$ & 8456 & 865 \\
\hline VIOLENCIA INTRAFAMILLRQ & $93 \%$ & $81 \%$ & $100 \%$ & $86 x$ \\
\hline CONTRAVENCIONES PENALISS & 713 & & & $31 \mathrm{~N}$ \\
\hline PENAL (PATROCINIO A VICTIMLS) & 85\% & $86 \%$ & $100 \%$ & 80 \\
\hline TERRAS & & $1000 \%$ & & $100 \%$ \\
\hline CIVIL & & 848 & $100 \%$ & $65 \%$ \\
\hline AGULAS & & & & $0 \%$ \\
\hline TOTAL & $87 \%$ & iss & $82 \%$ & $65 \%$ \\
\hline
\end{tabular}

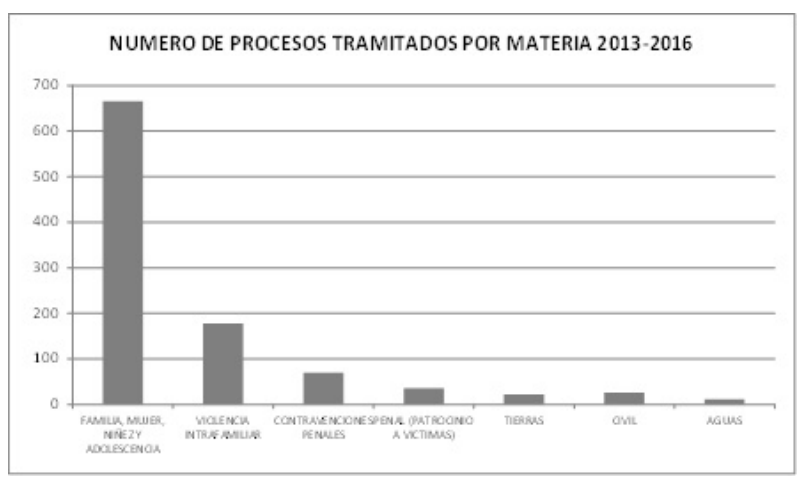

Figura 1. Fuente: Ficha de registro de usuarios atendidos en el Consultorio Jurídico Elaborado por:Los Autores

En cuanto al tipo de servicio jurídico (materia) que recibieron los usuarios que asistieron al consultorio jurídico en el período 2013-2015 de un total de 942 casos, el área relacionada con familia, mujer, niñez y adolescencia es la más representativa con 611 procesos $(65 \%)$, el indicador de cumplimiento promedio de procesos ganados es del $86 \%$; aquí se han atendido controversias de paternidad y alimentos, fijación de pensiones alimenticias, incidentes de pensión, régimen de visitas, tenencia, patria potestad, divorcios, disolución y liquidación de la sociedad conyugal. Los asuntos de violencia intrafamiliar ocupan el segundo lugar con 173 casos (18\%) del total de casos analizados y cuyo indicador de cumplimiento de los casos ganado frente al total es del $86 \%$ en este grupo se tratan conflictos de violencia contra la mujer y miembros del grupo familiar. El porcentaje restante $17 \%$ corresponden a conflictos en las materias: contravenciones penales, penal, tierra, civil y aguas, cuyos indicadores de casos ganados en promedio superan el $80 \%$.

El índice de procesos ganados por área es alto particularmente en las materias relacionadas con violencia intrafamiliar, mujer y familia en dónde de cada 100 casos alrededor de 86 son procesos que se han ganado. 
c) Indicador de audiencias fallidas frente al total de procesos tramitados

Tabla 4

Audiencias fallidas vs número total de procesos tramitados por años 2013-2015. indicador de casos fallidos Fuente: Ficha de registro de usuarios atendidos en el Consultorio Jurídico

Elaborado por: Los Autores

\begin{tabular}{|l} 
INDICADOR DE CASOS FALLIDOS \\
\begin{tabular}{|l|c|c|c|c|}
\hline \multicolumn{1}{|c|}{ AÑOS } & $\mathbf{2 0 1 3}$ & $\mathbf{2 0 1 4}$ & $\mathbf{2 0 1 5}$ & TOTAL \\
\hline PORCENTAJE DE CUMPLIMIENTO & $9 \%$ & $3 \%$ & $2 \%$ & $\mathbf{5 \%}$ \\
\hline NUMERO DE AUDIENCIAS FALLIDAS & 25 & 16 & 3 & $\mathbf{4 4}$ \\
\hline TOTAL PROCESOS & 291 & 464 & 187 & $\mathbf{9 4 2}$ \\
\hline
\end{tabular}
\end{tabular}

El indicador de audiencias fallidas que ocurren por la inasistencia, desacuerdo entre las partes o por ser materia no conciliable, en el período de análisis se ubica en el $5 \%$ del total de procesos, este indicador se ha ido reduciendo del $9 \%$ en el 2013 al $2 \%$ en el 2015. Apenas 5 de cada 100 audiencias no se han llevado a cabo.

\subsubsection{Indicadores sociales-económicos relacionados con} los usuarios que asisten al Consultorio Jurídico

a) Indicador de accesibilidad por lugar de procedencia

\section{Tabla 5}

Fuente: Ficha de registro de usuarios atendidos en el Consultorio Jurídico Elaborado por: Los Autores

\begin{tabular}{|c|c|c|c|c|c|}
\hline & & & & & \\
\hline & & AÑOS & & & \\
\hline LUGAR DE PROCEDENCIA & 2013 & 2014 & 2015 & TOTAL & $\%$ \\
\hline CANTON CAÑAR & 335 & 432 & 189 & 956 & $89 \%$ \\
\hline PARROQUIA URBANA & 257 & 284 & 121 & 662 & $69 \%$ \\
\hline AREA URBANA & 12 & 26 & 7 & 45 & $7 \%$ \\
\hline AREA RURAL PERIFERICA & 245 & 258 & 114 & 617 & $93 \%$ \\
\hline PARROQUIAS RURALES & 78 & 148 & 68 & 294 & $31 \%$ \\
\hline CHOROCOPTE & 9 & 15 & 3 & 27 & $9 \%$ \\
\hline INGAPIRCA & 19 & 30 & 17 & 66 & $22 \%$ \\
\hline CHONTAMARCA & 12 & 23 & 6 & 41 & $14 \%$ \\
\hline HONORATO VASQUEZ & 10 & 22 & 6 & 38 & $13 \%$ \\
\hline JUNCAL & 6 & 8 & 5 & 19 & $6 \%$ \\
\hline DUCUR & 0 & 10 & 10 & 20 & $7 \%$ \\
\hline GUALLETURO & 12 & 15 & 5 & 32 & $11 \%$ \\
\hline GENERAL MORALES & 8 & 12 & 2 & 22 & $7 \%$ \\
\hline SAN ANTONIO DE PAGUANCAY & 0 & 2 & 2 & 4 & $1 \%$ \\
\hline VENTURA & 0 & 2 & 0 & 2 & 196 \\
\hline OTRAS COMUNIDADES & 2 & 9 & 12 & 23 & $8 \%$ \\
\hline OTROS CANTONES & 7 & 68 & 46 & 121 & $11 \%$ \\
\hline TOTAL GENERAL & 342 & 500 & 235 & 1.077 & $100 \%$ \\
\hline
\end{tabular}

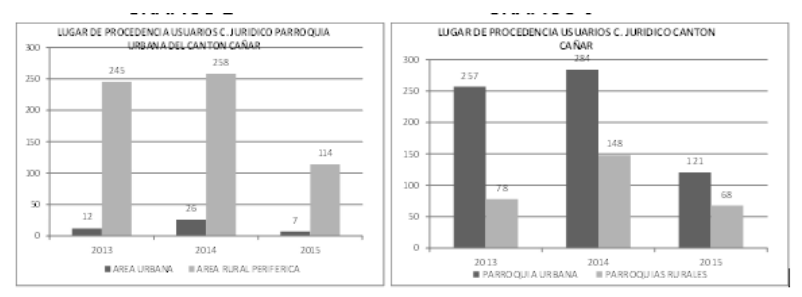

Figura 2. Fuente: Ficha de registro de usuarios atendidos en el Consultorio Jurídico Elaborado por: Los Autores

El porcentaje más alto por lugar de origen corresponde a la parroquia urbana del cantón Cañar que se ubica en un
$69 \%$ del total de usuarios que asistieron en el período de estudio al Consultorio Jurídico, esto es 662 personas, sin embargo de este total el $93 \%$ (617 casos) corresponden al área periférica rural, esto es comunidades campesinoindígenas como son Quilloac, San Rafael, La Posta, La Capilla, Lluillan, Quinuapata, Nar, entre otras. Una explicación a este comportamiento es de que los habitantes del centro urbano se encuentran en una mejor situación económica y prefieren acudir a un abogado privado o también por los prejuicios que tienen de acudir a una defensoría gratuita.

No menos importante con el $31 \%$ (294 casos) del total de casos atendidos son personas de parroquias rurales, provenientes de parroquias distantes como Ingapirca, Chontamarca y General Morales; estos dos últimos con población en su mayoría indígena.

Se aprecia para este indicador que el $84 \%$ de las personas que acuden al Consultorio Jurídico tienen como lugar de origen el área rural del cantón Cañar y que en su mayoría corresponden a comunidades de las áreas periféricas aledañas al centro urbano con características de población campesino-indígena.

\section{b) Indicador de género e identificación étnica}

Tabla 6

Fuente: Ficha de registro de usuarios atendidos en el Consultorio Jurídico Elaborado por: Los Autores
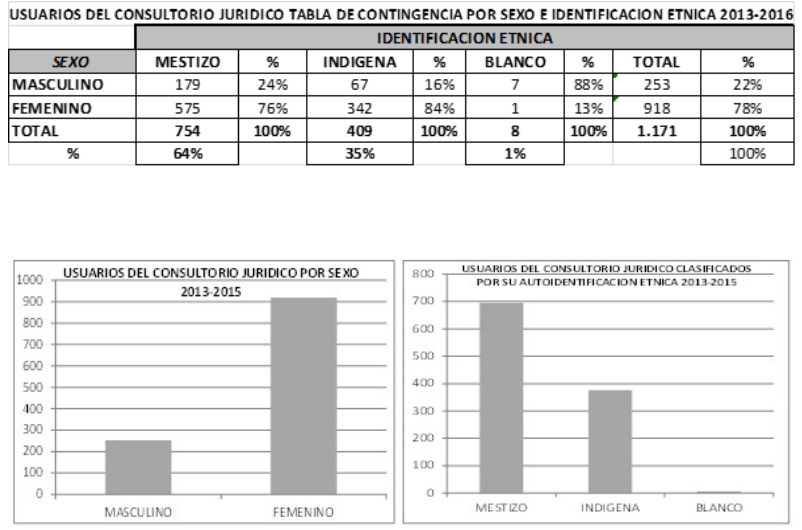

Figura 3. Fuente: Ficha de registro de usuarios atendidos en el Consultorio Jurídico Elaborado por: Los Autores

De los 1171 casos (1009 procesos y 162 asesorías) desde 2013 hasta abril de 2016, el $78 \%$ (918 personas) son del sexo femenino y de ellas el $35 \%$ (342 personas) se auto-identifican como indígenas; los casos más recurrentes que se presentan en este segmento se dan por violencia intrafamiliar, maltrato psicológico y físico, paternidad y alimentos, fijación de pensiones alimenticias, régimen de visitas y tenencia.

El indicador de género es alto de cada 10 personas que asisten al Consultorio Jurídico 8 son del sexo femenino y de las zonas rurales del cantón. 
El indicador de identificación étnica refiere que cada 10 personas que asisten al Consultorio Jurídico 6 de ellos se auto-identifican como mestizos y 4 se auto-identifican como indígenas.

c) Indicador de nivel de instrucción y actividad económica (ocupación)

Tabla 7

Fuente: Ficha de registro de usuarios atendidos en el Consultorio Jurídico Elaborado por: Los Autores

USUARIOS DEL CONSULTORIO JURDICO TABLA DE CONTINGENCAA POR INSTRUCCIÓN Y OCUPACION $2013-2016$

\begin{tabular}{|c|c|c|c|c|c|c|c|c|c|c|}
\hline & \multicolumn{10}{|c|}{ INSTRUCCIÓN } \\
\hline OCUPACION & NOESTUDIO & $\%$ & PRIMARIA & $\%$ & SECUNDARIA & $\%$ & SUPERIOR & $\%$ & TOTAL & $\%$ \\
\hline AMA DE CASA & 38 & $45 \%$ & 222 & $38 \%$ & 115 & $26 \%$ & 9 & $16 \%$ & 384 & $33 \%$ \\
\hline EMPLEADA DOMESTICA & 6 & $7 \%$ & 52 & $9 \%$ & 26 & $6 \%$ & 0 & $0 \%$ & 84 & $7 \%$ \\
\hline ESTUDIANTE & & $0 \%$ & 2 & $0 \%$ & 89 & $20 \%$ & 6 & $10 \%$ & 97 & $8 \%$ \\
\hline AGRICULTOR (JORNAL EROS) & 25 & $29 \%$ & 150 & $26 \%$ & 101 & $23 \%$ & 2 & $3 \%$ & 278 & $24 \%$ \\
\hline EMPLEADO PUBLICO & & $0 \%$ & 2 & $0 \%$ & 5 & $1 \%$ & 7 & $12 \%$ & 14 & $1 \%$ \\
\hline EMPLEADO PRNADO & & $0 \%$ & 20 & $3 \%$ & 23 & $5 \%$ & 17 & $29 \%$ & 60 & $5 \%$ \\
\hline DESEMPLEADOS & 7 & $8 \%$ & 50 & $9 \%$ & 23 & $5 \%$ & 10 & $17 \%$ & 90 & $8 \%$ \\
\hline OTROS (CUENTA PROPIA) & 9 & $11 \%$ & 86 & $15 \%$ & 62 & $14 \%$ & 7 & $12 \%$ & 164 & $14 \%$ \\
\hline TOTAL & 85 & 1 & 584 & 1 & 444 & 1 & 58 & 1 & 1.171 & 1 \\
\hline$\%$ & $7 \%$ & & $50 \%$ & & $38 \%$ & & $5 \%$ & & & $100 \%$ \\
\hline
\end{tabular}

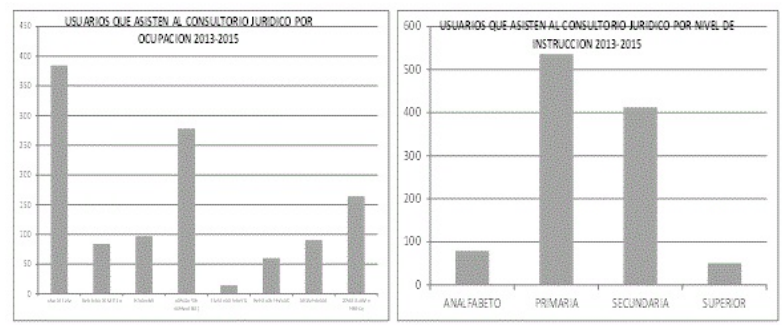

Figura 4. Fuente: Ficha de registro de usuarios atendidos en el Consultorio Jurídico Elaborado por: Los Autores

De acuerdo con el cuadro anterior el $57 \%$ del total de personas que han asistido al consultorio jurídico tienen educación de primaria o no han estudiado, de este grupo el $38 \%$ son amas de casa y el $30 \%$ se dedican a labores de agricultura en calidad de jornaleros/as y el $9 \%$ son empleadas domésticas.

Apenas un $5 \%$ tienen instrucción superior y que en su mayoría son empleados privados, y un $8 \%$ indica encontrase sin trabajo (desempleados).

El indicador del nivel de instrucción establece que de cada 10 usuarios que asisten al Consultorio Jurídico Gratuito 6 tienen un nivel de instrucción primaria o no han estudiado.

El indicador de actividad económica nos dice que de cada 10 personas que asisten al Consultorio Jurídico 3 son amas de casa y 2 son agricultores jornaleros en su mayoría con un bajo nivel educativo.

\section{d) Indicador de nivel de ingresos económicos por sexo}

Del total de usuarios que han asistido al Consultorio Jurídico en el período 2013-2015 más de la mitad ganan
Tabla 8

Fuente: Ficha de registro de usuarios atendidos en el Consultorio Jurídico Elaborado por: Los Autores

USUARIOS DEL CONSULTORIO JURIDICO TABLA DE CONTINGENCIA POR SEXO E INGRESOS 2013-2015

\begin{tabular}{|l|c|c|c|c|c|c|}
\cline { 2 - 8 } \multicolumn{1}{c|}{} & \multicolumn{9}{c|}{ SEXO } & \multicolumn{2}{c|}{ TOTAL GENERAL } \\
\hline INGRESOS & MASCULINO & $\%$ & FEMENINO & $\%$ & CANTIDAD & $\%$ \\
\hline MENOS DE 1 SBU & 139 & $58 \%$ & 489 & $98 \%$ & 628 & $58 \%$ \\
\hline 1 SBU & 92 & $38 \%$ & 316 & $63 \%$ & 408 & $38 \%$ \\
\hline 2SBU & 10 & $4 \%$ & 31 & $6 \%$ & 41 & $4 \%$ \\
\hline TOTAL & 241 & $100 \%$ & 836 & $167 \%$ & 1077 & $100 \%$ \\
\hline$\%$ & $22 \%$ & & $78 \%$ & & & $100 \%$ \\
\hline
\end{tabular}

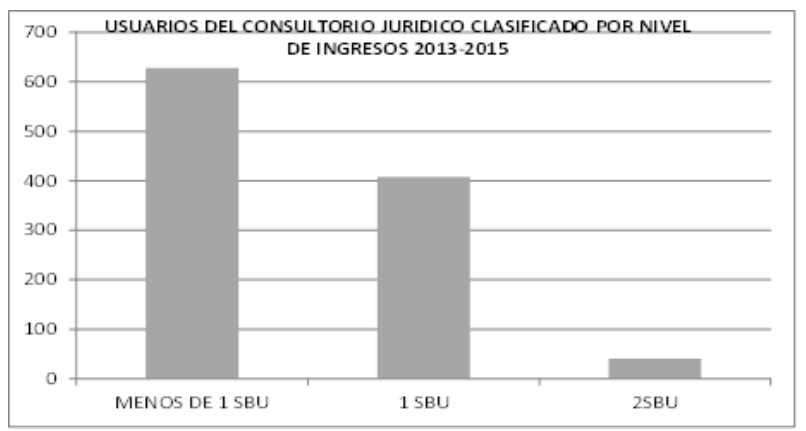

Figura 5. Fuente: Ficha de registro de usuarios atendidos en el Consultorio Jurídico Elaborado por: Los Autores

menos de un salario básico unificado y de este grupo el $78 \%$ son mujeres, apenas el $4 \%$ se encuentre entre 2 o más salarios básicos unificados.

El indicador de nivel de ingresos económicos por sexo nos dice que de cada 10 personas que han asistido al Consultorio Jurídico 9 perciben como ingresos al menos un salario básico unificado y 7 son mujeres.

\section{Conclusiones}

Luego del análisis de la información recabada se ha llegado a concluir lo siguiente:

1) El proyecto de vinculación con la comunidad "Consultorio Jurídico Gratuito" de la Universidad Católica de Cuenca extensión Cañar ha cumplido el objetivo de mejorar el acceso a la justicia y garantizar los derechos de la población del cantón Cañar y sus parroquias particularmente de aquellos que habitan en el área rural con altos niveles de pobreza, baja escolaridad; en su mayoría mujeres campesino-indígenas víctimas de violencia intrafamiliar y controversias relacionadas con la familia, niñez y adolescencia.

2) Lo niveles de eficiencia y efectividad en el tratamiento de los procesos y asesorías evidenciados en los altos indicadores relacionados con la atención y servicio al usuario ha demostrado que se ha cumplido con el objetivo planteado en el proyecto de vinculación.

3) El proyecto ha respondido a los estándares y lineamientos de género e interculturalidad para la educación superior al crear instancias específicas de denuncia de violencia intrafamiliar y en la aplicación concreta para 
la igualdad en el acceso a la justicia de pueblos y nacionalidades.

\section{Recomendaciones}

1) Con el propósito de obtener mayor información sobre la atención que el Consultorio Jurídico es necesario aplicar encuestas de satisfacción y de esta manera conocer el punto de vista de los usuarios.

2) Paralelo a la asesoría legal se deben realizar programas de capacitación, la presentación de casos y promoción en las comunidades rurales.
3) Es necesario que se elaboren protocolos de atención para casos específicos de acuerdo a la materia o área del derecho.

\section{Referencias}

de Derechos Humanos, C. I. (2007). Acceso a la justicia para las mujeres víctimas de violencia en las Américas. OEA.

de Derechos Humanos, I. I. (2011). Manual autoformativo sobre acceso a la justicia y derechos económicos, sociales y culturales. C.R: EDISA.

Moya, R. (1999). Interculturalidad y Educación: Diálogo para la democracia en América Latina. Docutech.

Scott, J. (1993). El género: Una categoría útil para el análisis histórico. Centro Editor de América Latina.

Secretaría de Educación Superior, Ciencia, T. e. I. (2015). Construyendo igualdad en la educación superior. Monsalve Moreno.

Walsh, C. (2009). Interculturalidad, estado, sociedad. UASB/Abya-Yala.

Recibido: 20 de Junio de 2016

Aceptado: 24 de mayo de 2017

Correa, Wilson: Economista de la Universidad de Cuenca, Magister en Administración de Negocios Universidad Técnica del Norte-Ibarra, Especialista en Administración de Instituciones Financieras de la UTE, Diplomado Superior en Desarrollo y Género de la FLACSO, Diplomado Superior en Educación Universitaria por Competencias de la UDA, Docente de la Universidad Católica de Cuenca.

Andrade,Hernán: Abogado de los Tribunales de Justicia de la Universidad Católica de Cuenca, Magister en Derecho Civil y Procesal de la UTPL, Especialista en Docencia Universitaria de la Universidad Católica de Cuenca, Docente de la Universidad Católica de Cuenca. Correo electrónico: haandradee@ucacue.edu.ec 
